\title{
Does Hearing Thresholds Vary Across Different Blood Groups?
}

\author{
Archana Rai Sarve ${ }^{1}$ Megha K. ${ }^{2}$ Devika Hem³ \\ ${ }^{1}$ Audiologist and Speech Language Pathologist, Nitte Institute of \\ Speech and Hearing, Mangalore, Karnataka, India \\ ${ }^{2}$ Audiologist and Speech Language Pathologist, Gupta's Speech and \\ Hearing Center, Bengaluru, Karnataka, India \\ ${ }^{3}$ Audiologist and Speech Language Pathologist, BRC Kerala \\ Education Department, Kerala, India \\ J Health Allied Sci ${ }^{\mathrm{NU}}$ 2019;9:17-21
}

\begin{abstract}
Address for correspondence Archana Rai Sarve, MASLP, Nitte Institute of Speech and Hearing, Medical Sciences Complex, Deralakatte, Mangalore 575018, Karnataka, India (e-mail: archana.rai04@gmail.com).
\end{abstract}

Abstract
Keywords
- behavioral threshold
- pure tone audiometry
- ABO classification
- blood group

The current study aimed at identifying the relationship between the blood group and hearing thresholds. The objective of the study was to identify if there is a difference in the pure tone audiometric thresholds of individuals with different blood groups, and to identify if there is a difference between pure tone audiometric thresholds of males and females of the same blood group. A prospective study was performed involving 80 normal hearing individuals between the age of 18 and 40 years. All participants included in the study had normal hearing $(<25 \mathrm{dBHL})$ and were Rh+. Participants complaining or giving indications of any kind of cochlear or retrocochlear pathological conditions were excluded from the study. Participants were grouped based on their blood groups. Each group consisted of 20 participants. Thresholds were established for frequencies ranging from $125 \mathrm{~Hz}$ to $20 \mathrm{KHz}$. Descriptive statistical analysis was done for mean and standard deviation. Bonferroni post hoc analysis was carried out for multiple comparisons, and the results revealed that there was no statistically significant difference in thresholds observed among different blood groups.

\section{Introduction}

Sight, hearing, taste, touch, and smell are the five senses of a human being. Among these five, hearing is the sense which helps us to enjoy the sounds and conversations. Hearing is the process where we pick up sounds and attach it to the meaning. Scientifically it is the process in which sound travels in invisible waves created by the vibrations of particles. Human ear can process very quiet sounds as well as extremely loud sounds. Humans are capable of hearing frequencies between $20 \mathrm{~Hz}$ and $20,000 \mathrm{~Hz}$, and have a fairly narrow range of hearing compared to other species. This range is known as the audio or sonic range. Any difficulty or inability to hear the sounds in the audio range is termed as hearing impairment or hearing loss.

Blood is a body fluid in humans and other animals that delivers nutrients and oxygen to the cells and transports metabolic waste products away from those same cells. ${ }^{1}$ Blood is classified based on the presence and absence of antigens and antibodies on the surface of red blood cells (RBCs). Based on the antigen property of the blood, all human beings can be classified into four groups. ${ }^{2}$ These human blood groups are as follows: antigen $A$ with antibody B-group A, antigen B with antibody A-group B, antigen $A B$ with no antibodies-group $A B$, and antigen nil with antibody $A$ and $B$-group $O$. Blood group $\mathrm{O}$ is the most common blood group (37.12\%) followed by B (32.26\%) and $A$ (22.88\%); the least prevalent blood group is $A B(7.74 \%)$.

Effect of blood group on some of the aspects of otoacoustic emissions (OAEs) including magnitude were studied. OAE status is indicative of outer hair cell functioning in the cochlea. OAE amplitude was found to be reduced in women aged between 18 and 26 years having $\mathrm{O}+$ blood group. ${ }^{3}$ In individuals with $\mathrm{O}+$ blood group, noise-induced hearing loss (NIHL) was determined to be significantly more frequent which indicated that individuals with $\mathrm{O}$ blood group are more prone to develop 
NIHL. ${ }^{4}$ Assessment was done for spontaneous otoacoustic emissions (SOAEs), transient-evoked emissions (TEOAEs), and distortion-product otoacoustic emissions (DPOAEs) of positive blood groups among which individuals with $\mathrm{O}$ blood group were found to have fewer occurrence of SOAEs and showed reduced amplitude in some of the frequencies for DPOAE when compared to the individuals with blood group $B .^{5}$ OAE amplitude was found to be reduced in participants with blood group $O$ when compared to those with blood groups A, B, and AB. This finding was supportive of the hypothesis that individuals with different blood groups may have different OAE amplitude values. ${ }^{6}$ Along with monitoring the OAEs, it is also important that regular monitoring of the hearing thresholds be carried out to extensively monitor the hearing sensitivity. Studies have been focused on identifying the relation between blood group and OAE amplitude. However, there is a dearth in literature that identifies the relation between blood groups and the behavioral hearing thresholds. The present study could serve as a baseline to understand the relation between blood groups and the behavioral hearing thresholds.

\section{Aims and Objectives}

The present study aims to identify the relationship between different blood group and behavioral thresholds (pure tone audiometry [PTA]) among individuals within the age range of 18 to 40 years (young adults). The objectives of the study are to

- Identify if there is a difference in the PTA thresholds of individuals with different blood group.

- Explore the difference in the PTA thresholds across gender within a particular blood group.

- Identify the difference in the PTA thresholds between right and left ear of individuals within a particular blood group.

\section{Methods}

The study included 80 participants, who were further grouped according to their blood group. Four blood groups (A, B, O, and $A B)$ were considered for the study. Only RH-positive blood group was considered for the study. Each group consisted of 20 participants, with 10 males and 10 females. Participants were within 18 to 40 years of age and had accurate knowledge of their blood group. The participants had reported no history of ear infection, prolonged noise exposure, or major health problems. Participants with the history of middle ear pathology or inner ear pathology were excluded from the study. Each participant completed a detailed case history questionnaire prior to the threshold determination. All participants provided the information regarding their age, blood group, hearing, and medical history. Immittance was done to rule out middle ear pathology, PTA was performed at frequencies from $125 \mathrm{~Hz}$ to $20 \mathrm{kHz}$ in both the ears using AC 40 clinical audiometer with supra-aural headphones with appropriate calibration. The stimulus used for PTA was pure tones of specific octave frequencies (from $125 \mathrm{~Hz}$ to $20 \mathrm{kHz}$ ). Threshold determination was done using $1 \mathrm{db}$ step. Statistical analysis was done using SPSS version 17. MANOVA and Bonferroni post hoc analysis was used for statistical analysis.

\section{Results and Discussion}

The present study aims to identify the difference in behavioral thresholds of individuals with different blood groups, in males and females. Mean and standard deviation of the thresholds for each blood group was derived from descriptive statistics and is depicted in graphs 1 to 4 . Results of the study suggest that thresholds of right ear are better than the thresholds of left ear in both males and females. MANOVA test was administered on the data to observe variation between the groups. Results of the parametric test revealed statistically significant difference only for right ear $(1.830[57,108.106] ; p=0.04)$. There was no statistically significant difference for thresholds of left ear across the blood groups at $p>0.05$. Results indicate hearing thresholds of the right ear were better both in males and females; this can be attributed to the slight anatomical difference in the right auditory pathway. Bonferroni post hoc analysis was carried out for multiple comparisons and there was no statistically significant difference in behavioral thresholds of different blood groups. This may be because of the noninfluence of different blood group antigens on outer hair cell functioning. Although the role of antigens in outer hair cell development and differentiation of inner ear parts are well acknowledged, it has no impact on the overall functioning of the inner ear. The biological role of human blood group antigens in tissue functions remains controversial. ${ }^{7}$ However, there are no evidence-based studies to explain why there is no difference in behavioral thresholds in different blood groups even though there is a difference observed in studies based on OAE (-Figs. 1-4). 


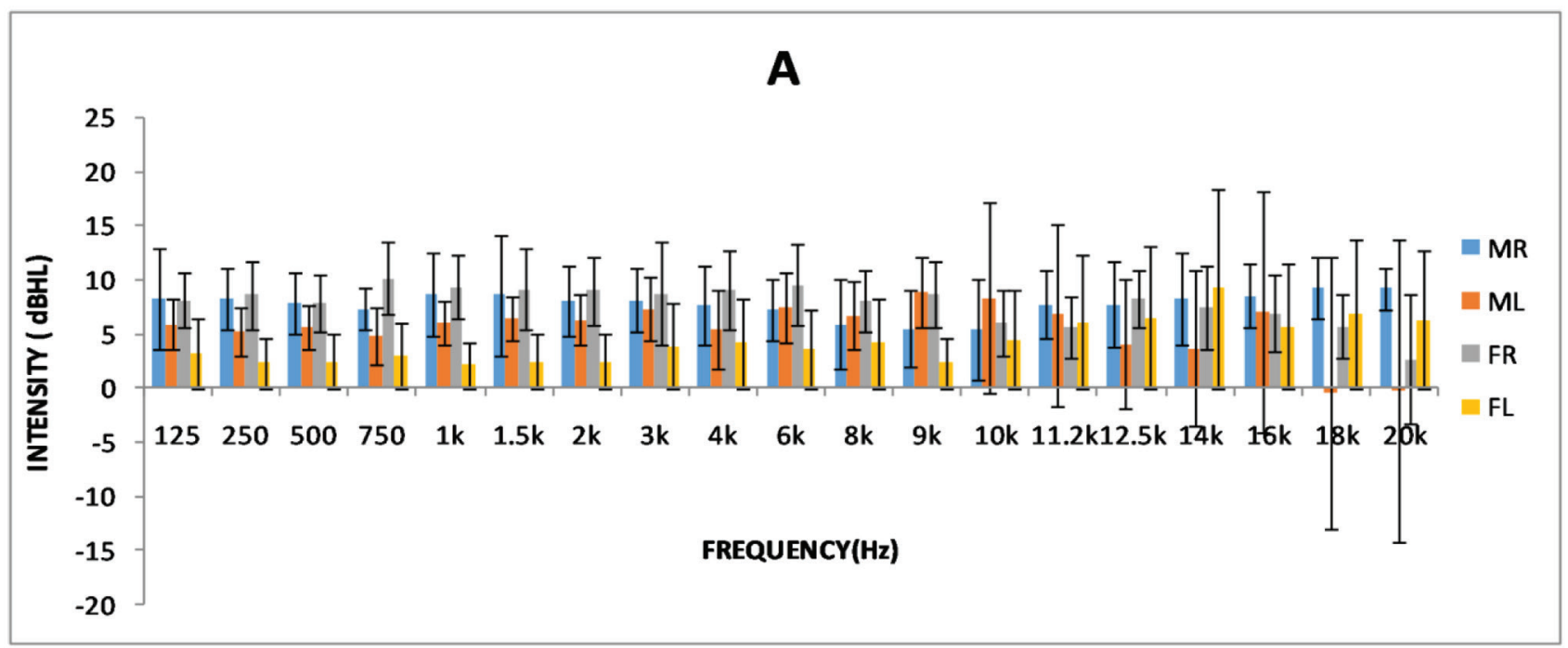

Fig. 1 Mean and SD of behavioral thresholds from $250 \mathrm{~Hz}$ to $20 \mathrm{KHz}$ in both ears for males and females (MR, male right; ML, male left; FR, female right; FL, female left) in blood group A. SD, standard deviation.

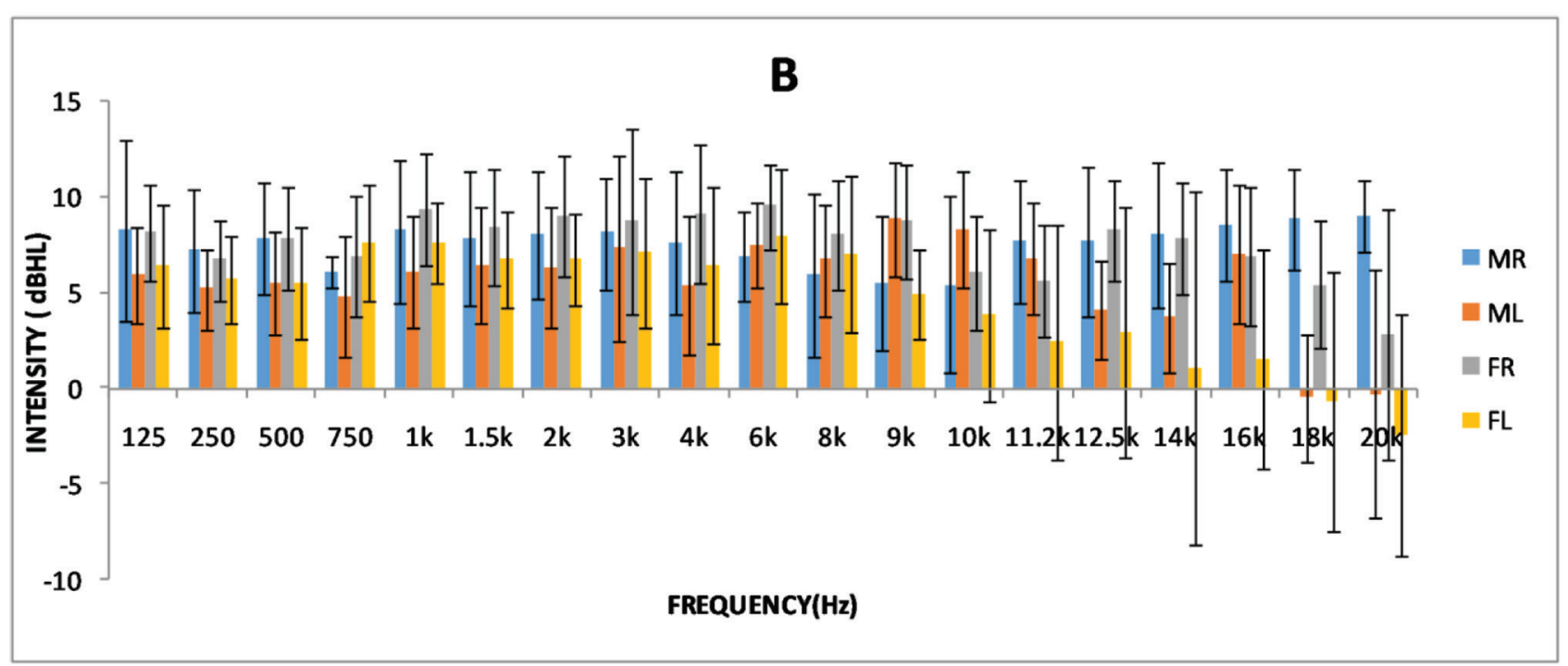

Fig. 2 Mean and SD of behavioral thresholds from $250 \mathrm{~Hz}$ to $20 \mathrm{KHz}$ in both ears for males and females (MR, male right; ML, male left; FR, female right; FL, female left) in blood group B. SD, standard deviation. 


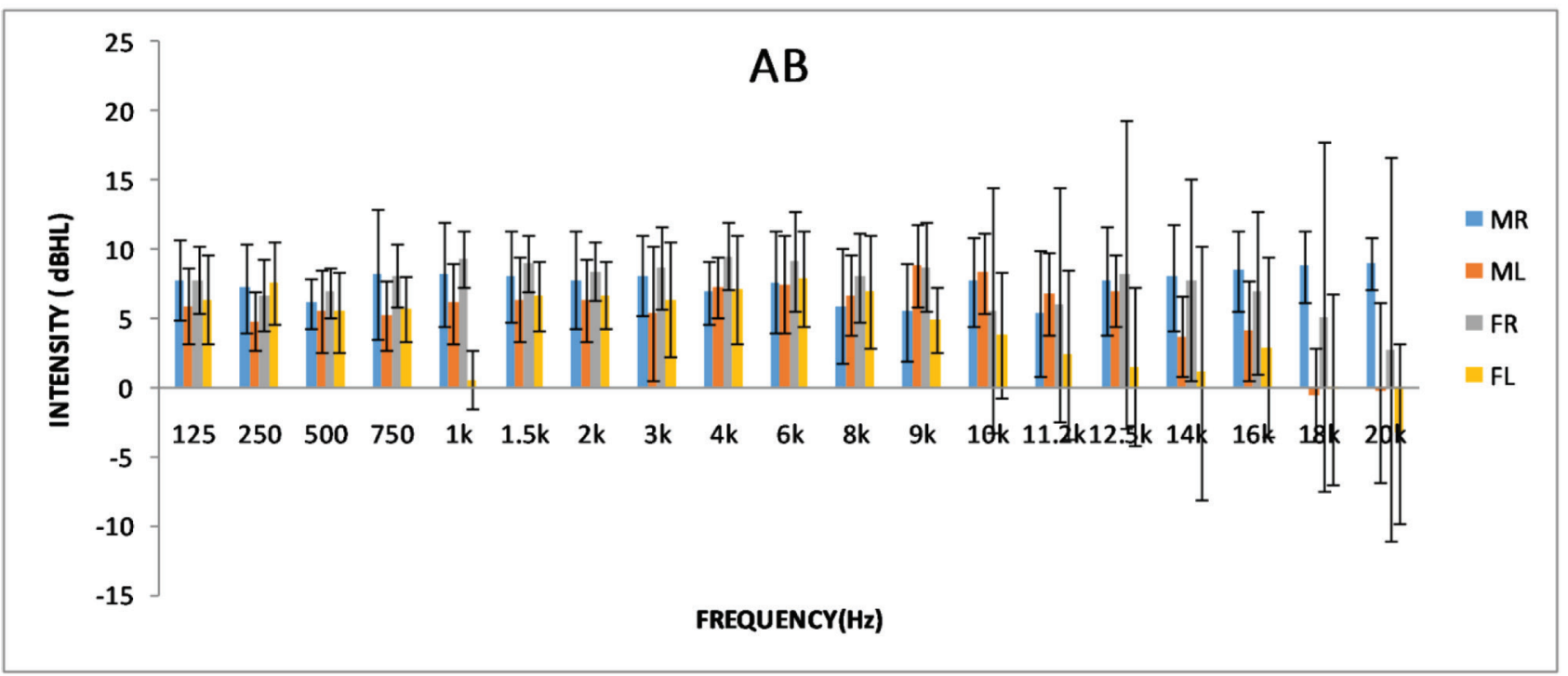

Fig. 3 Mean and SD of behavioral thresholds from $250 \mathrm{~Hz}$ to $20 \mathrm{KHz}$ in both ears for males and females (MR, male right; ML, male Left; FR, female right; $F L$, female left) in blood group $A B$. SD, standard deviation.

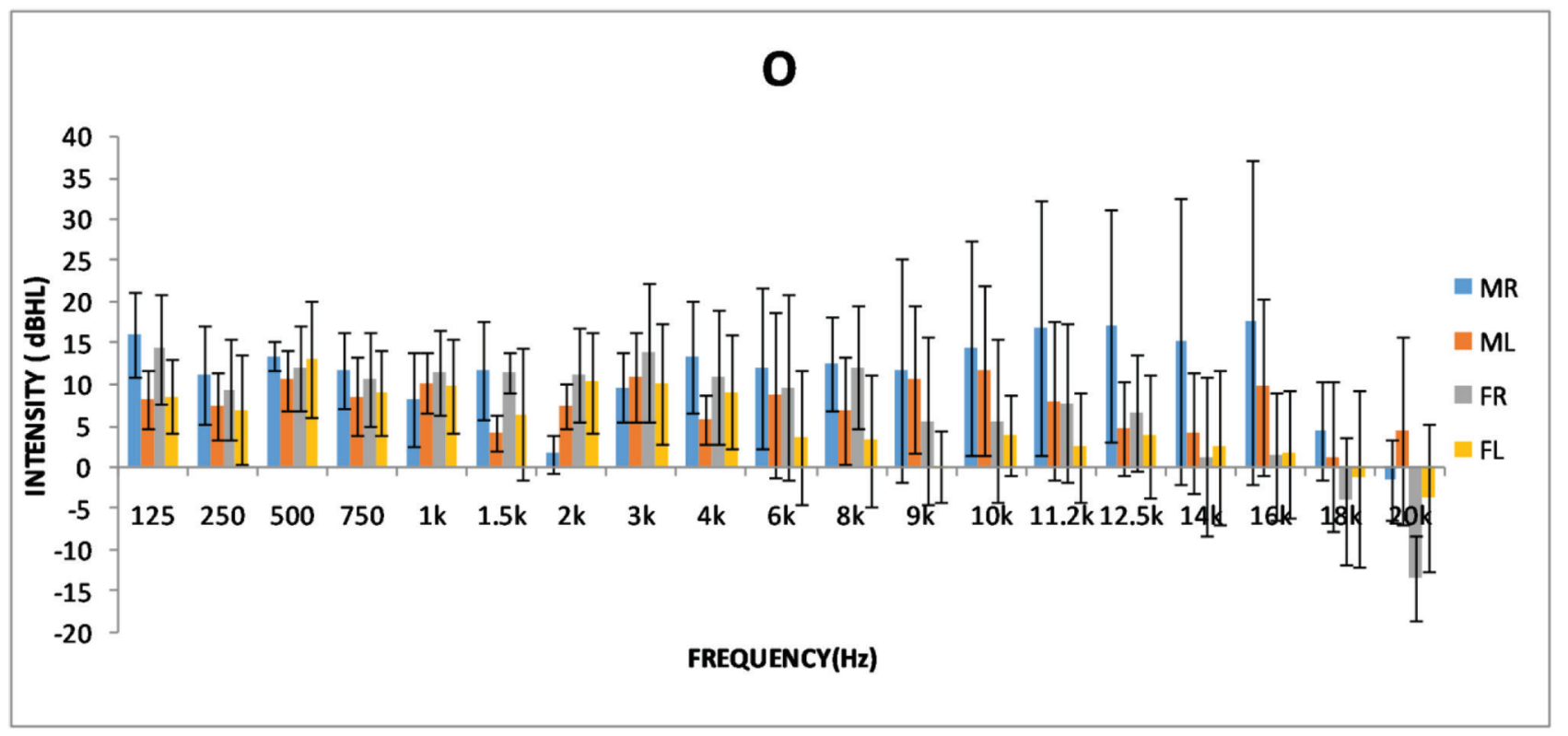

Fig. 4 Mean and SD of behavioral thresholds from $250 \mathrm{~Hz}$ to $20 \mathrm{KHz}$ in both ears for males and females (MR, male right; ML, male left; FR, female right; FL, female left) in blood group O. SD, standard deviation. 


\section{Conclusion}

The present study aimed to identify the difference in behavioral thresholds in different blood group in males and females. Findings reveal thresholds were better in right ear in males and females; however, there was no significant threshold difference noticed in a specific blood group, indicating that behavioral thresholds do not vary significantly among different blood groups. Further investigation on the potential link between ABO blood group and auditory status, including differential effects of noise exposure on cochlear function, is needed.

\section{Conflict of Interest}

None declared.

\section{Acknowledgment(s)}

The author(s) extend their gratitude to the Director, Nitte Institute of Speech and Hearing, for permitting us to carry out this research. We also thank the Principal, Nitte Institute of Speech and Hearing, for the constant support.

\section{References}

1 Mitra R, Mishra N, Rath GP. Blood groups systems. Indian J Anaesth 2014;58(5):524-528

2 Dean L. Blood Groups and Red Cell antigens. Bethesda (MD): National Center for Biotechnology Information; 2005

3 Chen WW, Chow KT, McPherson B. ABO blood group and cochlear status: otoacoustic emission markers. Ear Hear 2018;39(3):555-562

4 Doğru $H$, Tüz $M$, Uygur K. Correlation between blood group and noise-induced hearing loss. Acta Otolaryngol 2003;123(8):941-942

5 Chow KT, McPherson B, Fuente A. Otoacoustic emissions in young adults: effects of blood group. Hear Res 2016;333:194-200

6 Agarwal AC, Pujary K, Ganapathy K, Balakrishnan R, Nayak $\mathrm{DR}$, Hasan F. Pure tone audiometry and otoacoustic emissions for the assessment of hearing loss in diabetic patients. Indian Journal of Otology. 2013;19(1):13

7 Oriol R. Genetic control of the fucosylation of $A B H$ precursor chains. Evidence for new epistatic interactions in different cells and tissues. J Immunogenet 1990;17(4)-(5):235-245 